\title{
Search for light CP-odd Higgs decay with a charm tag at BABAR
}

\section{Georges Vasseur ${ }^{* \dagger}$}

CEA, IRFU, SPP, Centre de Saclay, Gif-sur-Yvette (FR)

E-mail: georges.vasseurecea.fr

\begin{abstract}
A search for a light CP-odd Higgs boson $A^{0}$ in $\Upsilon(1 S) \rightarrow \gamma A^{0}, A^{0} \rightarrow c \bar{c}$ decays is presented. The $\Upsilon(1 S)$ mesons are selected via the dipion transition $\Upsilon(2 S) \rightarrow \pi^{+} \pi^{-} \Upsilon(1 S)$. The $A^{0} \rightarrow c \bar{c}$ final state is tagged through the reconstruction of various $D^{(*)}$ mesons. No significant signal is observed, and limits on the product branching fraction $B\left(\Upsilon(1 S) \rightarrow \gamma A^{0}\right) \times B\left(A^{0} \rightarrow c \bar{c}\right)$ are established at the level of $7 \times 10^{-5}-2 \times 10^{-3}$ for $A^{0}$ mass values between $4.0 \mathrm{GeV} / c^{2}$ and $9.25 \mathrm{GeV} / c^{2}$.
\end{abstract}

The European Physical Society Conference on High Energy Physics

22-29 July 2015

Vienna, Austria

*Speaker.

On behalf of the BABAR collaboration. 


\section{Introduction}

A lot of extensions of the Standard Model predict a rich sector of Higgs bosons. In particular, a CP-odd Higgs boson might be light enough to be produced at B-factories in the radiative decay of the $\Upsilon$ resonances. That is why a campaign of comprehensive searches for a light Higgs boson is performed in $B A B A R$.

In the next to minimal supersymmetric standard model, for given parameters of the model, the branching ratio of $\Upsilon$ radiative decays to a CP-odd Higgs boson $A^{0}$ is predicted, as a function of the $A^{0}$ mass, to lie typically in the range from $10^{-7}$ to $10^{-4}$ [1]. If produced, the $A^{0}$ will decay into a fermion pair with a branching fraction proportional to the fermion mass square, so preferentially into the heaviest fermions kinematically allowed. Besides the down-type fermions are favored for large values of $\tan \beta$ and the up-type fermions for small values of $\tan \beta$. Figure 1 shows as a function of the $A^{0}$ mass the $A^{0}$ branching ratios into $c \bar{c}$ for different values of $\tan \beta$ [2]. As expected, the up-type $c \bar{c}$ mode is dominant between the $c \bar{c}$ kinematic threshold and the $b \bar{b}$ kinematic threshold for small values of $\tan \beta$.

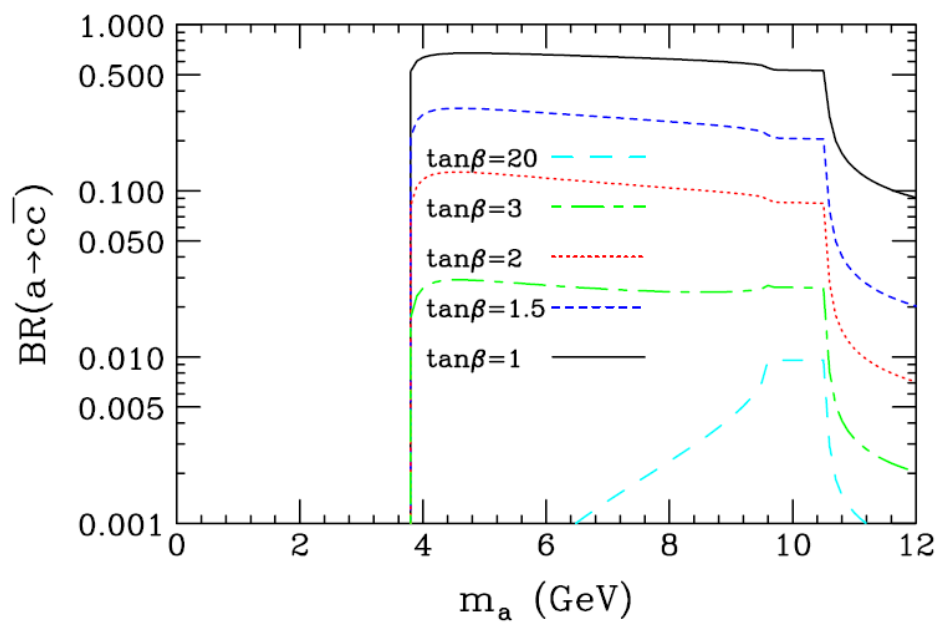

Figure 1: Predicted branching ratio of $A^{0} \rightarrow c \bar{c}$ as a function of the $A^{0}$ mass. The curves correspond to different values of $\tan \beta$.

The BABAR detector [3] has been in operation from 1999 to 2008 at SLAC on the PEP-II $e^{+} e^{-}$collider. Most data were taken at the $\Upsilon(4 S)$ resonance, above the $B$-meson pair threshold, to study $B$-meson physics and $\mathrm{CP}$ violation [4]. However during the last months of data taking, BABAR accumulated data on the $\Upsilon(3 S)$ and $\Upsilon(2 S)$ resonances. The analysis is based on the $\Upsilon(2 S)$ sample which consists of about $10^{8} \Upsilon(2 S)$ events and corresponds to a luminosity of $13.6 \mathrm{fb}^{-1}$. A luminosity of $1.4 \mathrm{fb}^{-1}$ was taken off-peak for background studies.

Previous searches for the $A^{0}$ have been performed at $B A B A R$ in various $A^{0}$ decay channels either in the radiative decays of the $\Upsilon(2 S)$ or $\Upsilon(3 S)$ resonances which produce a monoenergetic photon or in the $\Upsilon(2 S)$ or $\Upsilon(3 S)$ transitions to $\Upsilon(1 S) \pi^{+} \pi^{-}$followed by the radiative decay of the $\Upsilon(1 S)$ which can be tagged by the dipion system recoiling against the $\Upsilon(1 S)$. No analyses found a significant signal and upper limits have been obtained [5]. The $A^{0}$ decay into $c \bar{c}$ had not yet been used until the recent analysis from $B A B A R$ published this year [6]. 


\section{Analysis}

In this analysis, the event selection is done in the following way. Two oppositely charged pion candidates, whose recoiling mass is consistent within $10 \mathrm{MeV} / c^{2}$ with the $\Upsilon(1 S)$ mass, as shown in figure 2, are selected. An additionnal photon with at least $30 \mathrm{MeV}$ of energy is required. And finally the $c \bar{c}$ decay of the $A^{0}$ is tagged by reconstructing a $D^{0}, D^{+}$, or $D^{*+}$ meson in one of the five following modes: $D^{0} \rightarrow K^{-} \pi^{+}, D^{0} \rightarrow K^{-} \pi^{+} \pi^{+} \pi^{-}, D^{0} \rightarrow K_{S} \pi^{+} \pi^{-}, D^{+} \rightarrow K^{-} \pi^{+} \pi^{+}$or $D^{*+} \rightarrow \pi^{+} D^{0}\left(\rightarrow K^{-} \pi^{+} \pi^{0}\right)$.

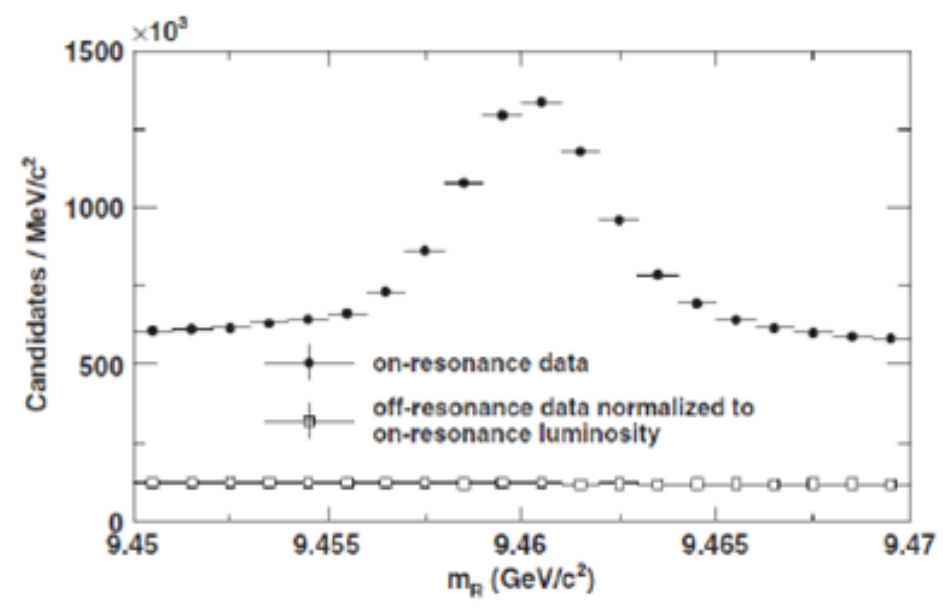

Figure 2: Dipion recoiling mass for (solid circles) on-resonance and (open squares) off-resonance data.

A further discrimination between signal and background is performed using a boosted decision tree using 24 input variables with kinematic, vertex, and event shape information. The main discriminating power comes from the kinematic variables.

The analysis is then based on the invariant mass $m_{X}$ of the hadronic system recoiling against the dipion and the photon, which is the Higgs mass for the signal searched for. As the background from low energy photons is a lot larger at high $m_{X}$, the analysis is divided into two overlapping regions, at low mass between 4 and $8 \mathrm{GeV} / c^{2}$ and at high mass between 7.5 and $9.25 \mathrm{GeV} / c^{2}$. Besides the region between 8.95 and $9.1 \mathrm{GeV} / c^{2}$ is excluded to avoid the transitions involving the $\chi_{b}$. After the selection, there remain respectively nearly $10^{4}$ candidates in the low mass region and $7.4 \times 10^{6}$ candidates in the high mass region, as illustrated in figure 3 . The main backgrounds are $\Upsilon(1 S) \rightarrow \gamma g g$ decays at low mass up to $7 \mathrm{GeV} / c^{2}$ and $\Upsilon(1 S)$ other decays above. The two other sources of background, $\Upsilon(2 S)$ decays without a $\pi^{+} \pi^{-}$transition to $\Upsilon(1 S)$ and $q \bar{q}$ continuum, account for about $15 \%$ of the background each.

It should be noted that the reconstructed $A^{0}$ width is expected to depend strongy on its mass, decreasing from about $120 \mathrm{MeV} / c^{2}$ at $4 \mathrm{GeV} / c^{2}$ to $8 \mathrm{MeV} / c^{2}$ at $9.25 \mathrm{GeV} / c^{2}$, as shown in figure 4 . This is because the photon energy resolution is better for lower photon energy corresponding to higher $A^{0}$ mass.

The overall reconstruction efficiency on the signal, including the $\mathrm{D}$ branching ratio, ranges from 4.0 to $2.6 \%$. The systematic uncertainty is about $10 \%$ and its main source is the $c \bar{c}$ hadronization. 

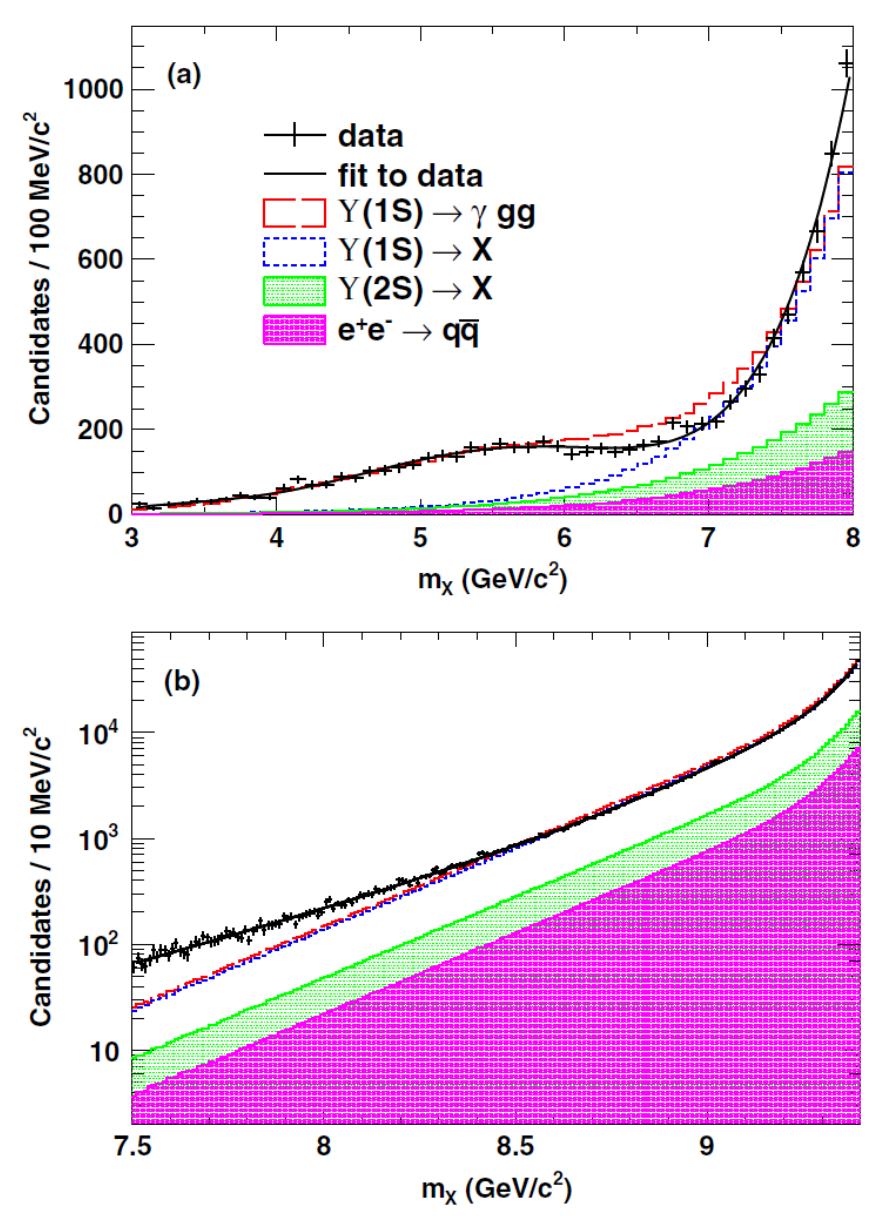

Figure 3: Recoiling mass against the dipion and photon in (a) the low mass region and (b) the high mass region. The points show the data and the histograms the cumulative background contributions.

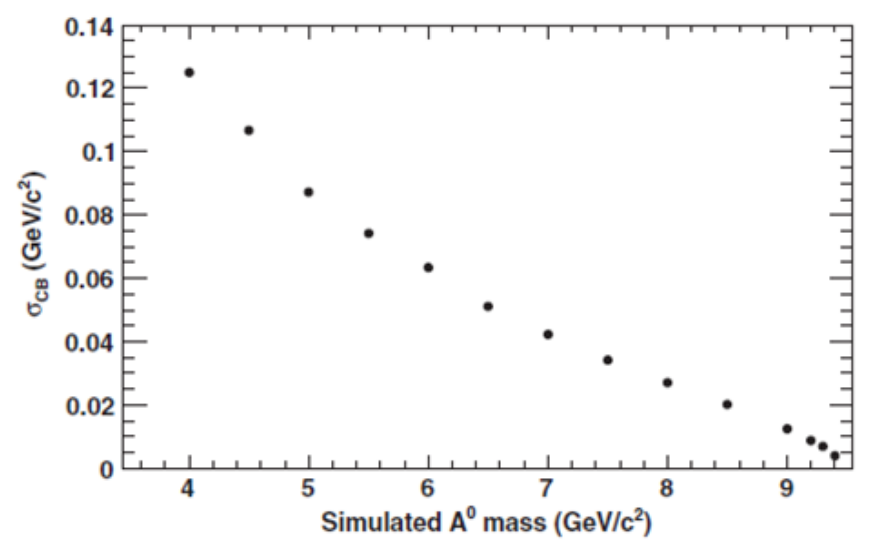

Figure 4: Reconstructed $A^{0}$ width as a function of $A^{0}$ mass.

The signal is extracted by performing extended maximum likelihood fits in local mass range in steps of $10 \mathrm{MeV} / c^{2}$ in the low mass region and steps of $2 \mathrm{MeV} / c^{2}$ in the high mass region. The signal PDF, whose parameters are fixed, is a Crystal Ball function. The background PDF, whose 

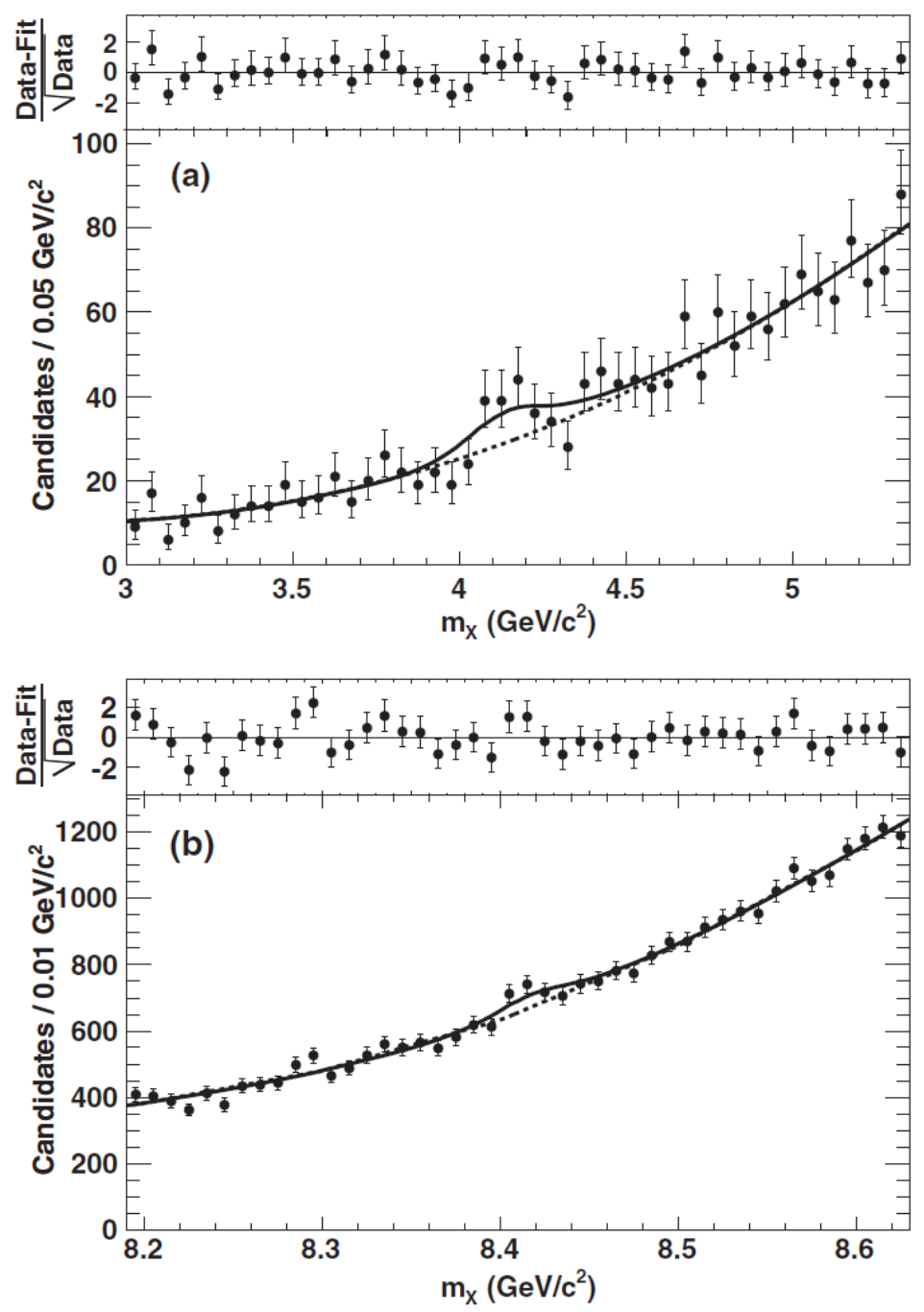

Figure 5: The fits in the recoiling mass against the dipion and photon with the highest local significance in (a) the low mass region and (b) the high mass region. The points show the data, the solid line is the fit allowing for a signal, and the dotted line is its background-only component.

parameters are free, is a second order polynomial. Figure 5 shows the fits with the highest local signal significance for the low mass region and the high mass region. The local signal significance never exceeds $2.3 \sigma$. Consequently no significant signal of CP-odd Higgs boson is seen for any value of the $A^{0}$ mass in the range under study.

\section{Result}

Therefore $90 \%$ confidence level upper limits are set on the product of branching ratios of $\Upsilon(1 S) \rightarrow \gamma A^{0}$ decays and of $A^{0} \rightarrow c \bar{c}$ decays. They are illustrated in figure 6 as a function of the $A^{0}$ mass and are in agreement with the expected limits. The upper limits obtained on the product of branching ratios range from $7.4 \times 10^{-5}$ to $2.4 \times 10^{-3}$ depending on the $A^{0}$ mass. 


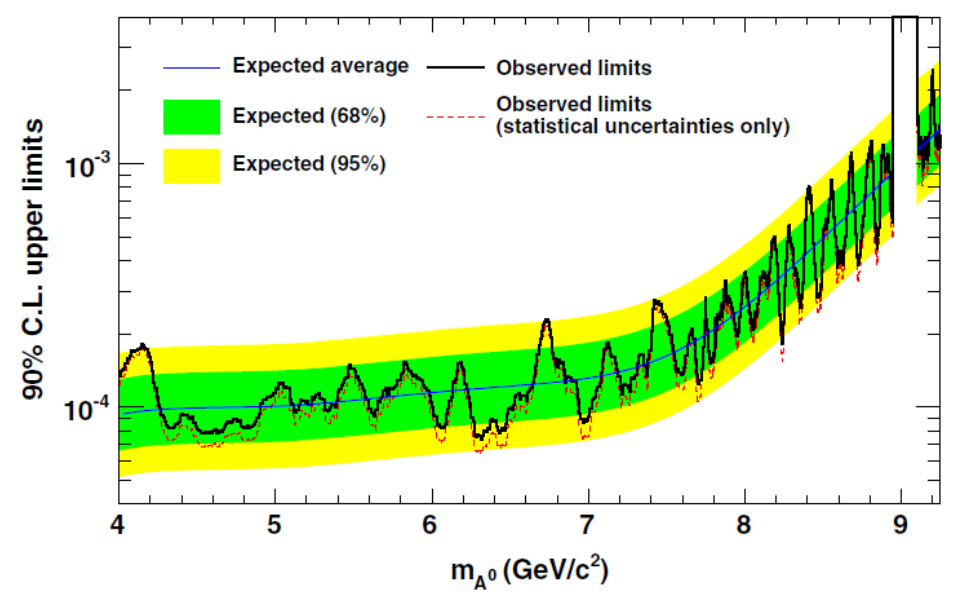

Figure 6: The 90\% confidence level upper limits on the product of the branching ratios $B\left(\Upsilon(1 S) \rightarrow \gamma A^{0}\right) \times$ $B\left(A^{0} \rightarrow c \bar{c}\right)$, shown by the black line as a function of the $A^{0}$ mass.

In summary, the B-factories can provide significant constraints on new physics models. In particular a low mass CP-odd Higgs boson has been searched for by the BABAR collaboration. A new channel, where the $A^{0}$ decays to $c \bar{c}$, has been explored. No significant signal has been found, as in all the many channels previously searched for. Upper limits have been set, constraining the $A^{0}$ production for a large fraction of the parameter space.

\section{References}

[1] R. Dermisek et al., Probing next-to-minimal-supersymmetric models with minimal fine tuning by searching for decays of the $\Upsilon$ to a light CP-odd Higgs boson, Phys. Rev. D 76, 051105 (2007).

[2] R. Dermisek and J.Gunion, New constraints on a light CP-odd Higgs boson and related NMSSM ideal Higgs scenarios, Phys. Rev. D 81, 075003 (2010).

[3] B. Aubert et al. (BABAR collaboration), The BABAR detector, Nucl. Instrum. Meth. A 729, 615 (2013).

[4] A.J. Bevan et al. (BABAR and Belle collaboration), The physics of the B factories, Eur. Phys. J. C 74, 3026 (2014).

[5] B. Aubert et al. (BABAR collaboration), Search for dimuon decays of a light scalar boson in radiative transitions $\Upsilon \rightarrow \gamma A^{0}$, Phys. Rev. Lett. 103, 081803 (2009); B. Aubert et al. (BABAR collaboration), Search for a low-mass Higgs boson in $\Upsilon(3 S) \rightarrow \gamma A^{0}, A^{0} \rightarrow \tau^{+} \tau^{-}$at BABAR, Phys. Rev. Lett. 103, 181801 (2009); J.P. Lees et al. (BABAR collaboration), Search for di-muon decays of a low-mass Higgs boson in radiative decays of the $\Upsilon(1 S)$, Phys. Rev. D 87, 031102 (2013); J.P. Lees et al. (BABAR collaboration), Search for a light Higgs boson decaying to two gluons or $\bar{s}$ in the radiative decays of $\Upsilon(1 S)$, Phys. Rev. D 88, 031701 (2013); J.P. Lees et al. (BABAR collaboration), Search for a low-mass scalar Higgs boson decaying to a $\tau$ pair in single-photon decays of $\Upsilon(1 S)$, Phys. Rev. D 88, 071102 (2013).

[6] J.P. Lees et al. (BABAR collaboration), Search for a light Higgs resonance in radiative decays of the $\Upsilon(1 S)$ with a charm tag, Phys. Rev. D 91, 071102 (2015). 